\title{
Depressive symptoms and apathy are associated with psychomotor slowness and frontal activation
}

\author{
Masayo Sawa $\cdot$ Hidehisa Yamashita • \\ Koichiro Fujimaki · Go Okada · Terumichi Takahashi • \\ Shigeto Yamawaki
}

Received: 28 July 2011/ Accepted: 25 January 2012/Published online: 10 February 2012

(C) The Author(s) 2012. This article is published with open access at Springerlink.com

\begin{abstract}
Affective symptoms, such as depression and apathy, and cognitive dysfunction, such as psychomotor slowness, are known to have negative impacts on the quality of life (QOL) of patients with mental and physical diseases. However, the relationships among depressive symptoms, apathy, psychomotor slowness, and QOL in a non-clinical population are unclear. The aim of the present study was to assess these relationships and examine the underlying cortical mechanisms in a non-clinical population. Fifty-two healthy male volunteers were assessed for depressive symptoms using the Zung Self-rating Depression Scale (SDS), for apathy measured using the Apathy Scale, and QOL using the Short-Form 36 item questionnaire (SF36). The volunteers also performed the Trail Making Test Part A (TMT-A) while undergoing assessment of hemoglobin concentration changes in the frontal cortical surface using 24-channel near-infrared spectroscopy (NIRS). The scores of the SDS and Apathy Scale showed significant negative correlations with the scores of
\end{abstract}

M. Sawa · T. Takahashi

Daijikai Mihara Hospital, 6-31-1 Nakano-cho,

Mihara, Hiroshima 723-0003, Japan

M. Sawa $\cdot$ H. Yamashita $\cdot$ G. Okada $\cdot$ S. Yamawaki $(\bowtie)$

Department of Psychiatry and Neurosciences,

Graduate School of Biomedical Sciences, Hiroshima University,

1-2-3 Kasumi, Minami-ku, Hiroshima 734-8551, Japan

e-mail: yamawaki@hiroshima-u.ac.jp

K. Fujimaki

Department of Occupational Therapy, Faculty of Health

and Welfare, Prefectural University of Hiroshima,

1-1 Gakuen-cho, Mihara, Hiroshima 723-0053, Japan

G. Okada

Department of Psychiatry, University of Michigan,

4250 Plymouth Road, Ann Arbor, MI 8109-2700, USA most of subscales of the SF36. In addition, the SDS score had a significant positive correlation with the time to complete the TMT-A. Further, activation of several frontal cortical areas had a significant positive correlation with the scores of the SDS and Apathy Scale. These results suggest that the degree of depressive symptoms and apathy are associated with a lower QOL in a non-clinical population and that cortical hyperactivation during a psychomotor task measured by NIRS may identify objectively individuals with a high degree of depressive symptoms and apathy.

Keywords Depressive symptoms · Apathy · Psychomotor slowness - Cortical activation - Quality of life

\section{Introduction}

Depressive symptoms and apathy have major impacts on the mental and physical health of individuals. Major depressive disorder (MDD), for example, is characterized by depressive symptoms and loss of interest, which is a component of apathy, and is a leading cause of worldwide disability. Worsening of depressive symptoms is associated with a reduced quality of life (QOL) [7, 17, 31]. The presence of subsyndromal depressive symptoms has also been shown to have a negative impact on psychosocial functioning [9]. In addition, there is increasing evidence that depressive symptoms are influential in the onset or progression of various kinds of diseases including Alzheimer's disease [25], coronary disease [11], and diabetes [1]. Furthermore, there is substantial evidence suggesting the negative impacts of depressive symptoms and apathy on QOL in many diseases including HIV [33], Parkinson's disease [21, 29], and brain tumors [14]. 
In addition to depressive symptoms and apathy, cognitive decline such as psychomotor slowness has a negative impact on social functioning of individuals. For example, Naismith et al. [20] reported that objectively measured psychomotor slowness is a significant predictor of physical disability in MDD, and Muslimovic et al. [19] reported that psychomotor slowness has a negative effect on QOL in Parkinson's disease. The Trail Making Test (TMT) is a popular neuropsychological instrument and is presumed to be a test of psychomotor skills [12, 27]. Functional neuroimaging studies have reported the involvement of the frontal cortical network in TMT [10, 18, 30, 40].

Meanwhile, neurocircuit abnormalities, an underlying condition in depressive symptoms and apathy in MDD, have been studied using neuroimaging approaches. For example, previous studies reported that anhedonic symptoms and depression severity were associated with reduced caudate volume [26] and decreased activation in the subgenual anterior cingulate cortex [16]. In addition, there is substantial evidence suggesting that psychomotor slowness in MDD is related to the fronto-striatal circuitry. Several studies using positron emission tomography (PET) reported that MDD patients with affective flattening and psychomotor slowness had decreased presynaptic dopamine function in the left caudate [2, 15].

In contrast to overt psychopathology such as MDD, there have been few studies that have examined the relationship among depressive symptoms, apathy and psychomotor slowness in a non-clinical population, and the cortical mechanisms of such symptomatology are unclear. Recently, the development of near-infrared spectroscopy (NIRS) has enabled non-invasive measurement of cortical activation under natural conditions, which enables examination while the subject performs a task related to psychomotor slowness such as the TMT-A. We hypothesized that the degree of depressive symptoms and apathy are associated with psychomotor slowness, as measured by TMT-A, and abnormal cortical activation, as measured by NIRS, as well as low QOL in a non-clinical population. We performed the following study to test this hypothesis directly.

\section{Methods}

\section{Subjects}

Fifty-two healthy male volunteers participated in this study (mean age, $37.4 \pm 11.1$ years). All subjects were determined to be right-handed using the Edinburgh Handedness Inventory scale [24]. Two experienced psychiatrists together excluded a participant with psychiatric symptoms above the threshold level. No subject had a history of major psychiatric disorder including major depressive disorder and anxiety disorder, neurological disorder, substance abuse, head injury, or major physical illness or was using any psychotropic medications at the time of the study. This study was approved by the Institutional Review Board of Mihara Hospital and the Prefectural University of Hiroshima. Written informed consent was obtained from each subject prior to the study.

Assessment of depressive symptoms, apathy, and QOL

Each subject was assessed for subjective depressive symptoms, extent of apathy, and QOL.

Subjective depressive symptoms were measured using the Zung Self-rating Depression Scale (SDS), a self-rating scale that consists of 20 questionnaires. The score of the SDS ranges from 20 (best) to 80 (worst), and the average is $35.1 \pm 8.0$ (mean $\pm \mathrm{SD}$ ) in the Japanese normal control population [5]. A higher score of the SDS is an indicative of a relatively greater degree of depressive symptoms.

Extent of apathy was measured using the Apathy Scale, a self-rating scale for assessing a tendency of apathy that consists of 16 questionnaires. The score of the Apathy Scale ranges from 0 (best) to 42 (worst), and the average is $8.7 \pm 6.6$ (mean $\pm \mathrm{SD}$ ) in the Japanese normal control population [23]. A higher score of the scale is an indicative of a relatively greater degree of apathy.

QOL was measured using the Medical Outcomes Study Short-Form 36-item questionnaire (SF36) [39]. SF36 is used widely to assess physical and mental well-being in social and individual contexts. Eight subscales are derived, referring to 8 health concepts: physical functioning (SF36-PF), role functioning-physical (SF36-RP), bodily pain (SF36-BP), general health (SF36-GH), vitality (SF36-VT), social functioning (SF36-SF), role functioning-emotional (SF36-RE), and mental health (SF36-MH). Each subscale ranges from 0 (worst health) to 100 (best health), and a score of 50 represents the mean score for the population.

\section{Activation task}

The activation task consisted of a 30 -s pre-task baseline, a TMT-A, and a 70-s post-task baseline. Each subject sat on a comfortable chair in a quiet room, and the subject was ordered to keep their head immobile as much as possible and to not speak. During the test, the subjects were required to draw a line as rapidly as possible joining consecutive numbers (1-25), which were pseudorandomly arranged on each page. We used series of 4 TMT-A sheets, which had different circle position patterns. The time required for completing the test (TMT time) was determined as a measure of task performance. During the pre-task and 
post-task periods, the subjects were instructed to draw lines repeatedly between two spots on a paper.

\section{NIRS measurement}

In this study, changes in [oxy-Hb] and [deoxy-Hb] were measured using a 24-channel NIRS machine (Hitachi ETG100) at two wavelengths of near-infrared light (i.e., 780 and $830 \mathrm{~nm})$. Absorption was measured, and [oxy-Hb] and [deoxy-Hb] were calculated. The distance between the pair of emission and detector probes was $3.0 \mathrm{~cm}$, and it was considered that the machine could measure points at a depth of $2-3 \mathrm{~cm}$ from the scalp, that is, the surface of the cerebral cortex [8, 35]. As shown in Fig. 1, the probes of the NIRS machine were placed on the subject's bilateral frontal region. The frontal probes measured hemoglobin concentration changes at 24 measurement points in a $6 \pm 15 \mathrm{~cm}$ area, with the lowest probes positioned along the Fp1-Fp2 line according to the international 10/20 system used in electroencephalography. The absorption of near-infrared light was measured with a time resolution of $0.1 \mathrm{~s}$. The obtained data were analyzed using the "integral mode". The pre-task baseline was determined as the mean across the last $10 \mathrm{~s}$ of the 30 -s pre-task period, and the post-task baseline was determined as the mean across the last $5 \mathrm{~s}$ of the 70-s post-task period. Linear fitting was applied to the data between these two baselines. The moving average method was used to exclude short-term motion artifacts in the analyzed data (moving average window: $5 \mathrm{~s}$ ).

\section{Data analyses}

The analysis focused on [oxy-Hb] changes. Changes in [oxy-Hb] were assumed to more directly reflect cognitive activation than [deoxy-Hb] changes, as shown by a

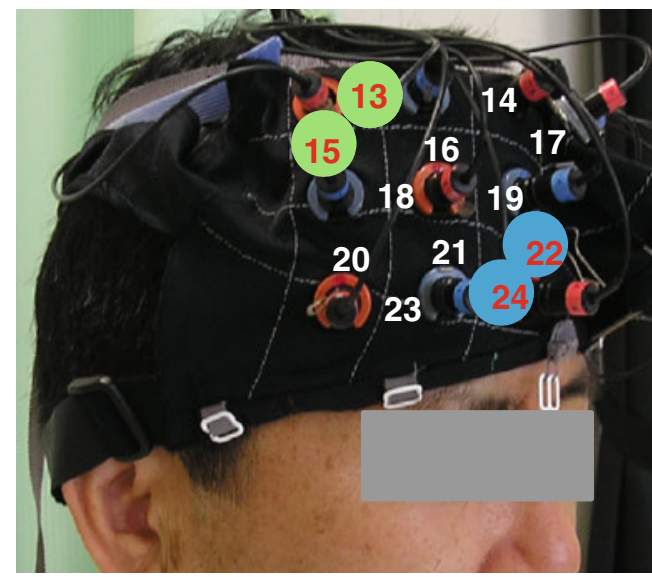

Fig. 1 Probe setting and channels showing significant correlations with the SDS and Apathy Scale. Yellow area indicates a channel showing significant correlations with the SDS. Blue areas indicate stronger correlation with blood-oxygenation level-dependent (BOLD) signals measured by fMRI [32].

NIRS data that clearly contained motion artifacts determined by a close observation of the subjects were excluded from analyses.

To examine the relationship among affective symptoms (SDS, Apathy Scale) and QOL (SF36), task performances (TMT time) and [oxyHb] changes during TMT, Pearson correlation analyses were conducted.

Statistical analysis was performed using PASW 18.0 software (Tokyo, Japan).

\section{Results}

Correlation between affective symptoms and QOL

Averaged scores of the SDS, Apathy Scale, and SF-36 are shown in Table 1. As shown in Table 2, the SDS negatively correlated with the SF36-RP $(r=-0.285, p=$ $0.041)$, SF36-BP $(r=-0.279, p=0.045), \mathrm{SF} 36-\mathrm{GH}(r=$ $-0.574, p<0.001)$, SF36-VT $(r=-0.635, p<0.001)$, SF36-RE $(r=-0.434, p=0.002)$, and SF36-MH $(r=$ $-0.640, p<0.001)$. The Apathy Scale negatively correlated with the SF36-PF $(r=-0.367, p=0.007)$, SF36GH $(r=-0.316, p=0.023)$, SF36-VT $(r=-0.459$, $p=0.001)$, SF36-RE $(r=-0.413, p=0.002)$, and SF36MH $(r=-0.433, p=0.001)$. These results suggest that depressive symptoms and apathy are closely related to a lower QOL.

Correlation between affective symptoms and task performance

The average TMT time was $75.4 \pm 18.3$ (mean \pm SD) seconds. The score of the SDS was positively correlated

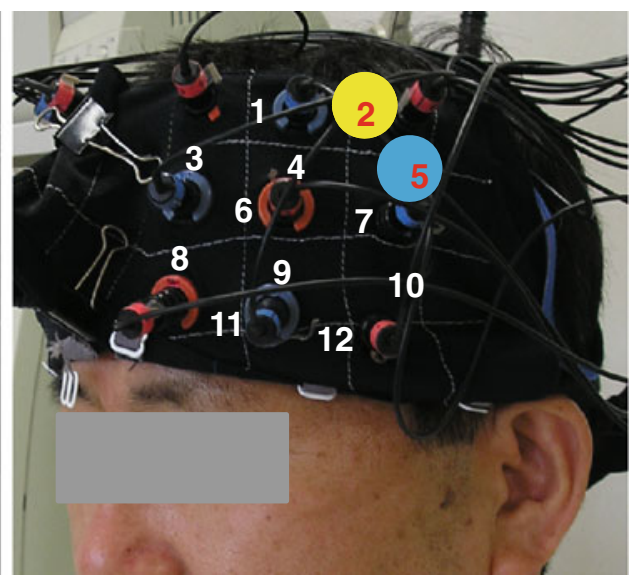

channels showing significant correlations with the Apathy Scale. Green areas indicate channels showing significant correlations with both the SDS and Apathy Scale 
Table 1 Affective symptoms and QOL

\begin{tabular}{lrc}
\hline & Mean & SD \\
\hline SDS & 36.8 & 7.7 \\
Apathy Scale & 9.8 & 6.0 \\
SF36-PF & 54.9 & 4. \\
SF36-RP & 50.5 & 7.0 \\
SF36-BP & 50.6 & 9.9 \\
SF36-GH & 50.9 & 10.9 \\
SF36-VT & 49.0 & 10.0 \\
SF36-SF & 50.7 & 8.5 \\
SF36-RE & 50.3 & 8.0 \\
SF36-MH & 49.4 & 9.2 \\
\hline
\end{tabular}

SD standard deviation, SDS Zung Self-rating Depression Scale, SF36 Medical Outcomes Study Short-Form 36-item questionnaire, $P F$ physical functioning, $R P$ role functioning, $B P$ bodily pain, $G H$ general health, $V T$ vitality, $S F$ social functioning, $R E$ role emotional, $M H$ mental health

Table 2 Correlation coefficients between affective symptoms and QOL

\begin{tabular}{lll}
\hline & SDS & Apathy Scale \\
\hline SF36-PF & -0.261 & $-0.367^{* *}$ \\
SF36-RP & $-0.285^{*}$ & -0.273 \\
SF36-BP & $-0.279^{*}$ & -0.207 \\
SF36-GH & $-0.574^{* *}$ & $-0.316^{*}$ \\
SF36-VT & $-0.635^{* *}$ & $-0.459^{* *}$ \\
SF36-SF & -0.189 & -0.218 \\
SF36-RE & $-0.434^{* *}$ & $-0.413^{* *}$ \\
SF36-MH & $-0.640^{* *}$ & $-0.433^{* *}$ \\
\hline
\end{tabular}

SDS Zung Self-rating Depression Scale, SF36 Medical Outcomes Study Short-Form 36-item questionnaire, $P F$ physical functioning, $R P$ role functioning, $B P$ bodily pain, $G H$ general health, $V T$ vitality, $S F$ social functioning, $R E$ role emotional, $M H$ mental health

$* p<0.05 ; * * p<0.01$

with TMT time $(r=0.357, p=0.009)$, suggesting that participants with depression took a longer time to complete the task. In contrast, there was no significant correlation between the score of the Apathy Scale and TMT time ( $r=0.261, p=0.062$ ).

Correlation between affective symptoms and [oxy-Hb] changes during task

As shown in Table 3 and Fig. 1, [oxy-Hb] changes during the TMT-A was positively correlated with SDS in $\mathrm{CH} 2$ ( $r=0.442, p=0.021)$, CH13 $(r=0.400, p=0.013)$, and CH15 $(r=0.528, p=0.006)$ and with Apathy Scale in CH5 $(r=0.451, p=0.046), \mathrm{CH} 13(r=0.372, p=$ $0.021)$, CH15 ( $r=0.0 .711, p<0.001), \mathrm{CH} 22(r=0.339$,
Table 3 Correlation coefficients between affective symptoms and [oxy-Hb] changes during TMT

\begin{tabular}{|c|c|c|}
\hline Channels & SDS & Apathy Scale \\
\hline 1 & 0.38 & 0.28 \\
\hline 2 & $0.442 *$ & 0.36 \\
\hline 3 & 0.28 & -0.05 \\
\hline 4 & 0.09 & 0.09 \\
\hline 5 & 0.18 & $0.451 *$ \\
\hline 6 & -0.09 & 0.29 \\
\hline 7 & -0.06 & -0.02 \\
\hline 8 & 0.21 & 0.19 \\
\hline 9 & -0.01 & 0.06 \\
\hline 10 & -0.30 & 0.17 \\
\hline 11 & 0.11 & 0.22 \\
\hline 12 & -0.06 & 0.21 \\
\hline 13 & $0.400 *$ & $0.372 *$ \\
\hline 14 & -0.04 & -0.12 \\
\hline 15 & $0.528 * *$ & $0.711 * *$ \\
\hline 16 & -0.02 & 0.23 \\
\hline 17 & 0.12 & 0.22 \\
\hline 18 & -0.09 & -0.01 \\
\hline 19 & 0.06 & 0.15 \\
\hline 20 & -0.10 & 0.08 \\
\hline 21 & -0.14 & 0.07 \\
\hline 22 & 0.14 & $0.339 *$ \\
\hline 23 & -0.07 & 0.16 \\
\hline 24 & 0.06 & $0.361 * *$ \\
\hline
\end{tabular}

SDS Zung Self-rating Depression Scale

$* p<0.05 ; * * p<0.01$

$p=0.017)$, and $\mathrm{CH} 24(r=0.361, p=0.009)$. No channel showed [oxy-Hb] changes during the TMT-A that were negatively correlated with the SDS or the Apathy Scale. These results suggest that participants with depression and apathy required greater levels of functional activation in several brain areas to complete the task.

\section{Discussion}

In this study, we demonstrated that depressive symptoms and apathy negatively affect brain function and QOL in a non-clinical population. An unexpected, but interesting result was that depressive symptoms had a greater negative impact on task performance than apathy. In this study, we showed that the score of the SDS was positively correlated with the TMT time, but the degree of apathy was not correlated with the TMT time. We also showed that participants with a high degree of depressive symptoms and apathy had a greater [oxy-Hb] increase in many frontal cortical regions. 
The degree of depressive symptoms and apathy were associated with most of indices of the SF-36. Our results are consistent with those reported by McCall et al. [17], who showed that an increasing severity of depression was associated consistently with worse QOL in MDD. Our results are also consistent with those of Oguru et al. [21], who reported that both the Apathy Scale and Beck Depression Inventory scores were negatively correlated with QOL in Parkinson's disease. Together, our results suggest that the presence of depressive symptoms and apathy has a negative impact on individual QOL.

The degree of depressive symptoms was associated significantly with psychomotor slowness, but the degree of apathy was not related to psychomotor slowness. The relationship between psychomotor slowness and age has been shown in previous studies [3, 34]. In our study, age was positively correlated with the TMT time, but there was no correlation between age and affective symptoms (data not shown). Psychomotor slowness in MDD has been shown in previous studies. For example, slower response times in MDD were observed on the TMT, Rule Shift Cards, and Stroop test [6]. Our results are consistent with those reported by Rosenberg et al. [28], who showed that the Geriatric Depression Scale was associated with incident impairment on all cognitive tests including the TMT-A in healthy older women. However, our results are inconsistent with those reported by Feli et al. [4], who showed that apathy correlated with a measure of information processing speed (Stroop test B) in older MDD patients. The reason for this inconsistency is unclear, but one possible reason is a difference between the tasks for psychomotor slowness. The TMT-A may not be sufficiently sensitive to detect the effects of apathy on brain function.

We also showed that participants with high degree of depressive symptoms and apathy had a greater [oxy-Hb] increase in many frontal cortical regions. Previous neuroimaging studies on cognitive impairment in MDD have demonstrated brain activation patterns with hypo-(e.g., Okada et al. [22]) and hyper-(e.g., Walter et al. [38]) activation of frontal cortical regions [13, 37]. In such studies, performance must be taken into account before attempting interpretation, and hyperactivation in context of equal or poorer performance is usually interpreted as 'inefficiency'. In this study, we found hyperactivation in the context of equal or poorer performance with a high degree of depressive symptoms and apathy, that is, inefficiency. Our results are consistent with those of Wagner et al. [36], who reported prefrontal hyperactivation with equal performance of the Stroop test in MDD using fMRI, and with results of Walter et al. [38], who reported that prefrontal hyperactivation with poor performance of Working Memory task in MDD using fMRI. Our results suggest that participants with a high degree of depressive symptoms and apathy require greater cortical resources to perform the same task. Furthermore, lower QOL and psychomotor slowness caused by depressive symptoms and apathy may be related to such inefficient frontal activation.

Our results are inconsistent with our hypothesis. We found that apathy was associated with low QOL and frontal cortical inefficiency, but was not correlated with psychomotor slowness. One potential explanation is that the effects of apathy may be more sensitively measured by cortical [oxy-Hb] changes detected by NIRS than by behavioral output. Thus, our present methods combining behavioral and NIRS measurement enabled us to detect the effects of apathy on brain function that would be difficult to detect by behavioral output alone.

There are several limitations in this study that should be taken into consideration. First, the participants were all male because women can have potentially influential factor such as mood fluctuations across the menstrual cycle, and our findings may not be generalizable to a female population. Second, assessments of depressive symptoms and apathy are based on self-rating scales without a structured diagnostic interview (e.g., SCID). Third, age and IQ were not controlled. They are potential factors capable of affecting not only psychomotor slowness, but also brain function and QOL. Fourth, depressive symptom was measured using the SDS. Although the SDS was developed specifically for patients with a diagnosis of major depression, the SDS is commonly used even in healthy subject study, since the scale is simple and less burdensome for subjects. Fifth, power analysis and multiple comparisons were not conducted in our study as in most previous NIRS studies. Further studies should take these factors into account. With these limitations in mind, this study provides evidence to support the hypothesis that depressive symptoms and apathy were associated with psychomotor slowness and abnormal cortical activation, as well as low QOL in a non-clinical population.

In conclusion, the degree of depressive symptoms and apathy were associated with lower QOL, and participants with high degree of depressive symptoms and apathy have inefficient cortical activations. On the basis of the findings, we assume that cortical hyperactivation during a psychomotor task measured by NIRS may be used to identify objectively individuals with a high degree of depressive symptoms and apathy. Further functional neuroimaging studies focusing on depressive symptoms and apathy at a non-clinical level may elucidate the brain mechanisms underlying depressive symptoms and apathy. These studies may be beneficial for promoting the QOL of healthy subjects and patients suffering from depressive symptoms and apathy. 
Acknowledgments This study is supported in part by a Grant-inAid from the Japanese Ministry of Health, Labour and Welfare (H21KOKORO-general-002). The authors would like to thank S. Aoyama for helpful support of NIRS analysis.

\section{Conflict of interest None.}

Open Access This article is distributed under the terms of the Creative Commons Attribution License which permits any use, distribution, and reproduction in any medium, provided the original author(s) and the source are credited.

\section{References}

1. Anderson RJ, Freedland KE, Clouse RE, Lustman PJ (2001) The prevalence of comorbid depression in adults with diabetes: a meta-analysis. Diabetes Care 24:1069-1078

2. Bragulat V, Paillere-Martinot ML, Artiges E, Frouin V, Poline JB, Martinot JL (2007) Dopaminergic function in depressed patients with affective flattening or with impulsivity: [18f]fluoro1-dopa positron emission tomography study with voxel-based analysis. Psychiatry Res 154:115-124

3. Davies AD (1968) The influence of age on trail making test performance. J Clin Psychol 24:96-98

4. Feil D, Razani J, Boone K, Lesser I (2003) Apathy and cognitive performance in older adults with depression. Int J Geriatr Psychiatry $18: 479-485$

5. Fukuda K, Kobayashi S (1983) Sds manual, japanese version (nihon-ban sds shiyou-tebiki). Sankyodo, Kyoto

6. Gohier B, Ferracci L, Surguladze SA, Lawrence E, El Hage W, Kefi MZ, Allain P, Garre JB, Le Gall D (2009) Cognitive inhibition and working memory in unipolar depression. J Affect Disord 116:100-105

7. Gostautas A, Pranckeviciene A, Matoniene V (2006) Changes in depression and quality of life during inpatient treatment of depression. Medicina (Kaunas) 42:472-478

8. Hock C, Villringer K, Muller-Spahn F, Wenzel R, Heekeren H, Schuh-Hofer S, Hofmann M, Minoshima S, Schwaiger M, Dirnagl U, Villringer A (1997) Decrease in parietal cerebral hemoglobin oxygenation during performance of a verbal fluency task in patients with Alzheimer's disease monitored by means of nearinfrared spectroscopy (NIRS) - correlation with simultaneous RCBF-pet measurements. Brain Res 755:293-303

9. Judd LL, Paulus MP, Wells KB, Rapaport MH (1996) Socioeconomic burden of subsyndromal depressive symptoms and major depression in a sample of the general population. Am $\mathbf{J}$ Psychiatry 153:1411-1417

10. Kubo M, Shoshi C, Kitawaki T, Takemoto R, Kinugasa K, Yoshida H, Honda C, Okamoto M (2008) Increase in prefrontal cortex blood flow during the computer version trail making test. Neuropsychobiology 58:200-210

11. Lett HS, Blumenthal JA, Babyak MA, Sherwood A, Strauman T, Robins C, Newman MF (2004) Depression as a risk factor for coronary artery disease: evidence, mechanisms, and treatment. Psychosom Med 66:305-315

12. Lezak MD, Howieson DB, Loring DW, Hannay HJ, Fischer JS (2004) Neuropsychological assessment. Oxford University Press, New York

13. Linden DE (2008) Brain imaging and psychotherapy: methodological considerations and practical implications. Eur Arch Psychiatry Clin Neurosci 258(Suppl 5):71-75

14. Mainio A, Tuunanen S, Hakko H, Niemela A, Koivukangas J, Rasanen P (2006) Decreased quality of life and depression as predictors for shorter survival among patients with low-grade gliomas: a follow-up from 1990 to 2003. Eur Arch Psychiatry Clin Neurosci 256:516-521

15. Martinot M, Bragulat V, Artiges E, Dolle F, Hinnen F, Jouvent R, Martinot J (2001) Decreased presynaptic dopamine function in the left caudate of depressed patients with affective flattening and psychomotor retardation. Am J Psychiatry 158:314-316

16. Matthews S, Simmons A, Strigo I, Gianaros P, Yang T, Paulus M (2009) Inhibition-related activity in subgenual cingulate is associated with symptom severity in major depression. Psychiatry Res 172:1-6

17. McCall WV, Cohen W, Reboussin B, Lawton P (1999) Effects of mood and age on quality of life in depressed inpatients. J Affect Disord 55:107-114

18. Moll J, de Oliveira-Souza R, Moll FT, Bramati IE, Andreiuolo PA (2002) The cerebral correlates of set-shifting: an FMRI study of the trail making test. Arq Neuropsiquiatr 60:900-905

19. Muslimovic D, Post B, Speelman JD, Schmand B, de Haan RJ (2008) Determinants of disability and quality of life in mild to moderate Parkinson disease. Neurology 70:2241-2247

20. Naismith S, Hickie I, Ward PB, Turner K, Scott E, Little C, Mitchell P, Wilhelm K, Parker G (2002) Caudate nucleus volumes and genetic determinants of homocysteine metabolism in the prediction of psychomotor speed in older persons with depression. Am J Psychiatry 159:2096-2098

21. Oguru M, Tachibana H, Toda K, Okuda B, Oka N (2010) Apathy and depression in Parkinson disease. J Geriatr Psychiatry Neurol 23:35-41

22. Okada G, Okamoto Y, Morinobu S, Yamawaki S, Yokota N (2003) Attenuated left prefrontal activation during a verbal fluency task in patients with depression. Neuropsychobiology 47:21-26

23. Okada K, Kobayashi S, Yamagata S, Takahashi K, Yamaguchi S (1997) Poststroke apathy and regional cerebral blood flow. Stroke 28:2437-2441

24. Oldfield RC (1971) The assessment and analysis of handedness: the Edinburgh inventory. Neuropsychologia 9:97-113

25. Ownby RL, Crocco E, Acevedo A, John V, Loewenstein D (2006) Depression and risk for Alzheimer disease: systematic review, meta-analysis, and metaregression analysis. Arch Gen Psychiatry 63:530-538

26. Pizzagalli DA, Holmes AJ, Dillon DG, Goetz EL, Birk JL, Bogdan R, Dougherty DD, Iosifescu DV, Rauch SL, Fava M (2009) Reduced caudate and nucleus accumbens response to rewards in unmedicated individuals with major depressive disorder. Am J Psychiatry 166:702-710

27. Reitan RM (1955) The relation of the trail making test to organic brain damage. J Consult Psychol 19:393-394

28. Rosenberg PB, Mielke MM, Xue QL, Carlson MC (2010) Depressive symptoms predict incident cognitive impairment in cognitive healthy older women. Am J Geriatr Psychiatry 18:204-211

29. Schrag A (2006) Quality of life and depression in Parkinson's disease. J Neurol Sci 248:151-157

30. Shibuya-Tayoshi S, Sumitani S, Kikuchi K, Tanaka T, Tayoshi S, Ueno S, Ohmori T (2007) Activation of the prefrontal cortex during the trail-making test detected with multichannel nearinfrared spectroscopy. Psychiatry Clin Neurosci 61:616-621

31. Starkstein SE, Mayberg HS, Preziosi TJ, Andrezejewski P, Leiguarda R, Robinson RG (1992) Reliability, validity, and clinical correlates of apathy in Parkinson's disease. J Neuropsychiatry Clin Neurosci 4:134-139

32. Strangman G, Culver JP, Thompson JH, Boas DA (2002) A quantitative comparison of simultaneous bold FMRI and NIRS recordings during functional brain activation. Neuroimage 17:719-731 
33. Tate D, Paul RH, Flanigan TP, Tashima K, Nash J, Adair C, Boland R, Cohen RA (2003) The impact of apathy and depression on quality of life in patients infected with HIV. AIDS Patient Care STDS 17:115-120

34. Tombaugh TN (2004) Trail making test A and B: normative data stratified by age and education. Arch Clin Neuropsychol 19:203-214

35. Toronov V, Webb A, Choi JH, Wolf M, Michalos A, Gratton E, Hueber D (2001) Investigation of human brain hemodynamics by simultaneous near-infrared spectroscopy and functional magnetic resonance imaging. Med Phys 28:521-527

36. Wagner G, Sinsel E, Sobanski T, Kohler S, Marinou V, Mentzel HJ, Sauer H, Schlosser RG (2006) Cortical inefficiency in patients with unipolar depression: an event-related FMRI study with the Stroop task. Biol Psychiatry 59:958-965
37. Walter H, Berger M, Schnell K (2009) Neuropsychotherapy: conceptual, empirical and neuroethical issues. Eur Arch Psychiatry Clin Neurosci 259(Suppl 2):S173-S182

38. Walter H, Wolf RC, Spitzer M, Vasic N (2007) Increased left prefrontal activation in patients with unipolar depression: an event-related, parametric, performance-controlled FMRI study. J Affect Disord 101:175-185

39. Ware JE Jr, Sherbourne CD (1992) The mos 36-item short-form health survey (sf-36). I. Conceptual framework and item selection. Med Care 30:473-483

40. Zakzanis KK, Mraz R, Graham SJ (2005) An FMRI study of the trail making test. Neuropsychologia 43:1878-1886 